\title{
Diffusive cosmic ray acceleration at relativistic shock waves
}

\author{
Reinhard Schlickeiser* ${ }^{\dagger}$ \\ Institut für Theoretische Physik, Lehrstuhl IV: Weltraum- und Astrophysik, Ruhr-Universität \\ Bochum, D-44780 Bochum, Germany \\ E-mail: rschetp 4 .rub.de
}

The analytical theory of diffusive cosmic ray acceleration at parallel stationary shock waves with magnetostatic turbulence is generalized to arbitrary shock speeds $V_{s}=\beta_{1} c$, including in particular relativistic speeds. This is achieved by applying the diffusion approximation to the relevant Fokker-Planck particle transport equation formulated in the mixed comoving coordinate system. In this coordinate system the particle's momentum coordinates $p$ and $\mu=p_{\|} / p$ are taken in the rest frame of the streaming plasma, whereas the time and space coordinates are taken in the observer's system. For magnetostatic slab turbulence the diffusion-convection transport equation for the isotropic (in the rest frame of the streaming plasma) part of the particle's phase space density is derived. For a step-wise shock velocity profile the steady-state diffusion-convection transport equation is solved. For a symmetric pitch-angle scattering Fokker-Planck coefficient $D_{\mu \mu}(-\mu)=D_{\mu \mu}(\mu)$ the steady-state solution is independent of the microphysical scattering details. For nonrelativistic mono-momentum particle injection at the shock the steady-state differential number density of accelerated particles is a Lorentzian-type distribution function which at large momenta approaches a power law distribution function $N\left(p \geq p_{c}\right) \propto p^{-\xi}$ with the spectral index $\xi\left(\beta_{1}\right)=1+\left[3 /\left(\Gamma_{1} \sqrt{r^{2}-\beta_{1}^{2}}-1\right)\left(1+3 \beta_{1}^{2}\right)\right]$. For nonrelativistic $\left(\beta_{1} \ll 1\right)$ shock speeds this spectral index agrees with the known result $\xi\left(\beta_{1} \ll 1\right) \simeq(r+2) /(r-1)$, whereas for ultrarelativistic $\left(\Gamma_{1} \gg 1\right)$ shock speeds the spectral index value is close to unity.

The 34th International Cosmic Ray Conference,

30 July- 6 August, 2015

The Hague, The Netherlands

\footnotetext{
* Speaker.

${ }^{\dagger}$ This work was partially supported by the Deutsche Forschungsgemeinschaft through grants Schl 201/23-1 and Schl 201/29-1.
} 


\section{Introduction}

Diffusive first-order Fermi acceleration at nonrelativistic shock fronts has been regarded as a prime candidate for particle acceleration in astrophysics[1,2]. Diffusive shock acceleration should also operate efficiently at magnetized shock waves with relativistic speeds. Such relativistic shocks form during the interaction of relativistic supersonic and super-Alfvenic outflows with the ambient ionized interstellar or intergalactic medium producing anisotropic counterstream plasma distribution functions due to shock-reflected charged particles in the upstream medium. Relativistic outflows are a direct consequence of violent explosive events such as in gamma-ray burst sources, but also occur as highly collimated pulsar winds and jets of active galactic nuclei with initial bulk Lorentz factors $\Gamma_{0}=\left(1-\left(V_{0} / c\right)^{2}\right)^{-1 / 2} \simeq 400$.

The transport and acceleration of energetic particles in the partially turbulent cosmic magnetic fields associated with shocks is described using the Fokker-Planck equation for the particle distribution function[3]. The diffusion approximation for the particle density in the rest frame of the fluid is a well-known simplified form of the Fokker-Planck equation, which results when turbulent pitch-angle scattering is strong enough to ensure that the scale of the particle density variation is signicantly greater than the particle mean free path. While for nonrelativistic shock waves the analytic theory of diffusive shock acceleration is well developed[4, 5, 6, 7], for relativistic shock speeds such an analytical theory does not exist sofar even for parallel shock waves, although the underlying Fokker-Planck transport equation (see Eq. (2.1) below) for the particle dynamics has already been derived[8,9] some years ago. Here we develop the analytical study of cosmic ray acceleration in parallel relativistic magnetized shock waves employing the diffusion approximation in the upstream and downstream regions of the shock wave. The development runs much in parallel with the existing work on nonrelativistic shocks.

\section{Relevant transport equations}

Magnetized space plasmas such as the interstellar medium harbour low-frequency linear ( $\delta B \ll$ $B_{0}$ ) transverse MHD waves (such as shear Alfven and magnetosonic plasma waves) with dispersion relations $\omega_{R}^{2}=V_{A}^{2} k_{\|}^{2}$ and $\omega_{R}^{2}=V_{A}^{2} k^{2}$, respectively, in the rest frame of the moving plasma. Faraday's induction law then indicates for MHD waves that the strength of turbulent electric fields $\delta E=\left(V_{A} / c\right) \delta B \ll \delta B$ is much smaller than the strength of turbulent magnetic fields. The ordering $B_{0} \gg \delta B \gg \delta E$ corresponds to the derivation of cosmic ray transport equations for $<f>(\vec{X}, p, \mu, \phi, t) \rightarrow f_{0}(\vec{X}, p, \mu, t) \rightarrow F(\vec{X}, p, t)$ from the collisionfree Boltzmann equation for the full phase space distribution $\langle f\rangle(\vec{X}, p, \mu, \phi, t)$ to the Fokker-Planck equation for its gyrotropic part $f_{0}(\vec{X}, p, \mu, t)$, and to the diffusion-convection transport equation for its isotropic part $F(\vec{X}, p, t)$, respectively.

When considering the acceleration of relativistic cosmic rays at relativistic shocks in the observer's frame of reference (laboratory frame), both the particles and the shock have relativistic speed. Consequently, the cosmic ray particles have a highly anisotropic gyrotropic velocity distribution due to the relativistic flow of the upstream and downstream medium in this laboratory frame. Therefore, most importantly, in order to employ the diffusion approximation, one has to study the cosmic ray dynamics instead in the mixed comoving coordinate system where the momentum co- 
ordinates of the cosmic rays $p$ and $\mu=p_{\|} / p$ are taken in the rest frame of the streaming plasma, and the time $t^{*}$ and space coordinates $z^{*}$ are taken in the laboratory (=observer) system. Chosing this mixed phase space coordinate system then automatically removes the strong particle momentum or velocity anisotropy due to the relativistic moving media. And we are allowed to employ the diffusion approximation to the gyrotropic cosmic ray distribution function: i.e. we approximate it by its isotropic part (in the rest frame of the flow!) and a small anisotropy.

Dropping from now on the $\left(z^{*}, t^{*}\right)$-notation (with the understanding that they have to be taken in the laboratory frame), the Larmor-phase averaged Fokker-Planck transport equation in a medium with magnetostatic turbulence only, propagating with the stationary bulk speed $\vec{U}=U(z) \vec{e}_{z}$ with $\Gamma=\left[1-\left(U^{2} / c^{2}\right)\right]^{-1 / 2}$ aligned along the uniform magnetic field $\left(\vec{B}_{0}=B_{0} \vec{e}_{z}\right)$ direction is given by $[8,9]$

$$
\begin{gathered}
\Gamma\left[1+\frac{U v \mu}{c^{2}}\right] \frac{\partial f_{0}}{\partial t}+\Gamma[U+v \mu] \frac{\partial f_{0}}{\partial z}-\alpha(z)\left(\mu+\frac{U}{v}\right)\left[\mu p \frac{\partial f_{0}}{\partial p}+\left(1-\mu^{2}\right) \frac{\partial f_{0}}{\partial \mu}\right]+\mathscr{R} f_{0}-S(\vec{X}, p, t) \\
=\frac{\partial}{\partial \mu}\left[D_{\mu \mu} \frac{\partial f_{0}}{\partial \mu}\right]
\end{gathered}
$$

where $\alpha(z)$ and $\mathscr{R} f_{0}$ denote the rate of adiabatic deceleration/acceleration in relativistic flows

$$
\alpha(z)=\frac{c^{2}}{U(z)} \frac{d \Gamma(z)}{d z}=\frac{d U}{d z} \Gamma^{3}=\frac{d(U \Gamma)}{d z}, \mathscr{R} f_{0}=-p^{-2} \frac{\partial}{\partial p}\left[p^{2} \dot{p}_{\text {loss }} f_{0}\right]+\frac{f_{0}}{T_{c}}
$$

and continuous ( $\left.\dot{p}_{\text {loss }}\right)$ and catastrophic $\left(T_{c}\right)$ momentum losses of cosmic ray particles, respectively.

For spatially constant flows the rate of adiabatic deceleration/acceleration (2.2) vanishes, and the remaining flow velocity $(U)$ dependent terms in Eq. (2.1) simply result from the Lorentz transformation of special relativity of the comoving-frame position-time coordinates $(z, t)$ to the laboratory-frame position-time coordinates $\left(z^{*}, t^{*}\right)$. However, for spatially varying flow speeds $U(z)$ special relativity no longer applies and has to be replaced by the transformation laws from general relativity. As noted[9, 10] these introduce connection coefficients or Christoffel symbols of the first kind. In a flat Euclidean space-time metric the terms proportional to $\alpha(z)$ in Eq. (2.1) are exactly these connection coefficients.

Due to the rapid pitch angle scattering the gyrotropic particle distribution function $f_{0}(\vec{X}, p, \mu, t)$ adjusts very quickly to a distribution function which is close to the isotropic distribution $F(\vec{X}, z, p, t)$ in the rest frame of the moving background plasma, i.e.

$$
f_{0}(\vec{X}, p, \mu, t)=F(\vec{X}, p, t)+g(\vec{X}, p, \mu, t), F(\vec{X}, p, t) \equiv \frac{1}{2} \int_{-1}^{1} d \mu f_{0}(\vec{X}, p, \mu, t),
$$

with $|g| \ll F$ and $\int_{-1}^{1} d \mu g(\vec{X}, p, \mu, t)=0$. Inserting the ansatz (2.3) into the Fokker-Planck equation (2.1) and averaging this equation over $\mu$, using that $D_{\mu \mu}(\mu= \pm 1)=0$, leads to the diffusionconvection transport equation

$$
\begin{gathered}
\Gamma\left[\frac{\partial F}{\partial t}+U \frac{\partial F}{\partial z}\right]-\frac{\alpha}{3} p \frac{\partial F}{\partial p}+\mathscr{R} F-S(\vec{X}, p, t)+\frac{v}{2} \Gamma\left(\frac{\partial}{\partial z}+\frac{U}{c^{2}} \frac{\partial}{\partial t}\right) \int_{-1}^{1} d \mu \mu g \\
-\frac{\alpha U}{2 v}\left[p \frac{\partial}{\partial p}+2\right] \int_{-1}^{1} d \mu \mu g-\frac{\alpha}{2}\left[p \frac{\partial}{\partial p}+3\right] \int_{-1}^{1} d \mu \mu^{2} g=0
\end{gathered}
$$


involving the first and second moment of the anisotropy. Subtracting Eq. (2.4) from the FokkerPlanck equation (2.1) provides a long and involved equation for the anisotropy $g$, which we approximate to leading order by

$$
\Gamma v \mu \frac{\partial F}{\partial z} \simeq \frac{\partial}{\partial \mu}\left[D_{\mu \mu} \frac{\partial g}{\partial \mu}\right]
$$

where we neglect the time derivate of $F$ as compared to the spatial gradient of $F$, i.e. $\left(U / c^{2}\right)(\partial F / \partial t) \ll$ $(\partial F / \partial z)$. Integrating Eq. (2.5) twice provides for the cosmic ray anisotropy

$$
g(\vec{X}, p, \mu, t) \simeq \frac{\Gamma v}{4}\left[\int_{-1}^{1} d \mu \frac{(1-\mu)\left(1-\mu^{2}\right)}{D_{\mu \mu}(\mu)}-2 \int_{-1}^{\mu} d x \frac{\left(1-x^{2}\right)}{D_{\mu \mu}(x)}\right] \frac{\partial F}{\partial z},
$$

which allows the calculation of the moments needed in the diffusion-convection transport equation (2.4) as

$$
\int_{-1}^{1} d \mu \mu g=-\frac{\Gamma \nu K_{0}}{4} \frac{\partial F}{\partial z}, \int_{-1}^{1} d \mu \mu^{2} g=-\frac{\Gamma \nu K_{1}}{6} \frac{\partial F}{\partial z}, K_{n}=\int_{-1}^{1} d \mu \frac{\mu^{n}\left(1-\mu^{2}\right)^{2}}{D_{\mu \mu}(\mu)}
$$

With these moments the diffusion-convection transport equation (2.4) becomes

$$
\Gamma \frac{\partial F}{\partial t}+\frac{\partial}{\partial z}\left[\Gamma\left(U F-\Gamma \kappa_{z z} \frac{\partial F}{\partial z}\right)\right]+\frac{1}{p^{2}} \frac{\partial}{\partial p}\left[p^{2} \kappa_{p z} \Gamma \frac{\partial F}{\partial z}-\frac{\alpha p^{3} F}{3}\right]+\mathscr{R} F=S(\vec{X}, p, t)
$$

with the two diffusion coefficients

$$
\kappa_{z z}=\frac{v^{2} K_{0}}{8}, \kappa_{p z}=\kappa_{z p}=\alpha K_{p z}, K_{p z}=\frac{v p}{12}\left(K_{1}+\frac{3 U}{2 v} K_{0}\right)
$$

Eq. (2.8) is the diffusion-convection transport equation of cosmic rays in aligned parallel flows of arbitrary speed containing magnetostatic slab turbulence with the cosmic ray phase space coordinates taken in the mixed comoving coordinate system. It is particularly appropriate to investigate cosmic ray particle acceleration in parallel relativistic flows.

In the limit of nonrelativistic flows $U(z) \ll c$ so that $\Gamma \simeq 1$. the transport equation (2.8) reduces to

$$
\frac{\partial F}{\partial t}+\frac{\partial}{\partial z}\left[U F-\kappa_{z z} \frac{\partial F}{\partial z}\right]+\frac{1}{p^{2}} \frac{\partial}{\partial p}\left[p^{2} \kappa_{p z} \frac{\partial F}{\partial z}-\frac{\alpha p^{3} F}{3}\right]+\mathscr{R} F=S(\vec{X}, p, t),
$$

which differs from the transport theory used in earlier nonrelativistic diffusive shock acceleration theory by the additional third last term on the left-hand side involving $\kappa_{p z}$, which results from our correct handling of the connection coefficients in Eq. (2.1). This additional term provides a major modification of the resulting steady-state differential momentum spectrum of accelerated particles in the nonrelativistic flow limit at nonrelativistic particles momenta: instead of a power law distribution of accelerated particles at the shock a Lorentzian distribution function results, which at large momenta then approaches the power law distribution inferred in earlier acceleration theories for nonrelativistic shock speeds. The modification to the Lorentzian at low particle momenta is due to the $\kappa_{p z}$-term in Eq. (2.8) because $\kappa_{p z} / p$ depends on the cosmic ray particle velocity and therefore is not constant at nonrelativistic particle momenta. 


\section{Steady-state particle acceleration at relativistic shock waves}

We adopt particle injection $S(\vec{X}, p, t)=S(p) \delta(z)$ at the position of the shock only, and adopt the step-like shock profile $U(z)=-U_{1}=$ const. for $0<z \leq \infty$ (upstream) and $U(z)=-U_{2}=$ const. for $-\infty<z \leq 0$ (downstream) with $U_{2}<U_{1}$, where we chose the rest frame of the shock wave as laboratory frame. In this case the rate of adiabatic acceleration (2.2)

$$
\alpha=\alpha_{0} \delta(z), \alpha_{0}=-\left(U_{1} \Gamma_{1}-U_{2} \Gamma_{2}\right)
$$

is non-zero only at the position of the shock. In the steady-state case with no losses $(\mathscr{R} \mathscr{F}=0)$ the diffusion-convection transport equation (2.8) in the rest frame of the shock wave then reduces to

$$
\frac{\partial}{\partial z}\left[\Gamma\left(U F-\Gamma \kappa_{z z} \frac{\partial F}{\partial z}\right)\right]+\frac{\alpha_{0} \delta(z)}{p^{2}} \frac{\partial}{\partial p}\left[p^{2} K_{p z} \Gamma \frac{\partial F}{\partial z}-\frac{p^{3} F}{3}\right]=S(p) \delta(z=0)
$$

For the up- and down-stream regions we obtain the solutions for spataially constant flow velocities and diffusion coefficients

$$
F_{1}(z>0, p)=F_{0}(p) \exp \left[-\frac{U_{1} z}{\Gamma_{1} \kappa_{z z, 1}}\right], F_{2}(z<0, p)=F_{0}(p)
$$

The particle momentum spectrum $F_{0}(p)$ of accelerated particles at the position of the shock is obtained by integrating the transport equation (2.9) from $z=-\eta$ to $z=\eta$ and considering the limit $\eta \rightarrow 0$. This provides the continuity condition for the cosmic ray streaming density at the shock. For a monomomentum injection spectrum $S(p)=S_{0} \delta\left(p-p_{0}\right)$ we obtain[11]

$$
F_{0}\left(p \geq p_{0}\right)=\frac{3 S_{0}}{U_{1} \Gamma_{1}-U_{2} \Gamma_{2}} \frac{p_{0}^{2}}{p^{2} T(p)} e^{-\psi I\left(p, p_{0}\right)}
$$

with the integral

$$
I\left(p, p_{0}\right)=\int_{\frac{m c}{p}}^{\frac{m c}{p_{0}}} \frac{d y}{y\left[1+\beta_{1} \sqrt{1+y^{2}}\left[Z R+3 \beta_{1} \sqrt{1+y^{2}}\right]\right.}
$$

and

$$
\psi=\frac{3}{\sqrt{\frac{r^{2}-\beta_{1}^{2}}{1-\beta_{1}^{2}}}-1}=\frac{3}{\Gamma_{1} \sqrt{r^{2}-\beta_{1}^{2}}-1}
$$

in terms of shock wave flow compression ratio $r=U_{1} / U_{2}=\beta_{1} / \beta_{2}$, the charge sign $Z=q /|q|$ of the cosmic ray particle and the helicity-dependent function

$$
R\left(s, \sigma_{+}, \sigma_{-}, H_{c}\right)=\frac{(2-s)(4-s)}{(3-s)(5-s)}\left[\sigma_{+}+\sigma_{-}+H_{c}\left(\sigma_{+}-\sigma_{-}\right)\right]
$$

The function $R$ arises for asymmetric pitch-angle scattering Fokker-Planck coefficients when we consider slab Alfven waves only with power-law type wave intensities $I \propto k_{\|}^{-s}$ and wavenumberindependent values of the cross helicity $H_{c}$ and the magnetic helicities $\sigma_{ \pm}$of forward and backward moving Alfven waves (isospectral turbulence). 
For the differential number density of accelerated particles $N(z, p)=4 \pi p^{2} F(z, p)$ we derive

$$
\begin{gathered}
N_{2}(z<0, p)=N_{0}\left(z=0, p \geq p_{0}\right)=4 \pi p^{2} F\left(p \geq p_{0}\right)=\frac{4 \pi S_{0} \psi}{U_{2} \Gamma_{2}} \frac{p_{0}^{2}}{T(p)} e^{-\psi I\left(p, p_{0}\right)}, \\
N_{1}(z>0, p)=N_{0}\left(z=0, p \geq p_{0}\right) \exp \left[-\frac{U_{1} z}{\Gamma_{1} \kappa_{z z, 1}}\right]
\end{gathered}
$$

\section{Results and discussion}

For a symmetric pitch-angle Fokker-Planck coefficients $(R=0)$ the integral (3.5) reduces to

$$
I(R=0)=\frac{1}{2\left(1+3 \beta_{1}^{2}\right)} \ln \frac{1+\frac{p^{2}}{p_{c}^{2}}}{1+\frac{p_{0}^{2}}{p_{c}^{2}}}, p_{c}\left(\beta_{1}\right)=\sqrt{\frac{3 \beta_{1}^{2}}{1+3 \beta_{1}^{2}}} m c,
$$

so that the differential number density at the shock (3.8) becomes the Lorentzian-type (or kappa) distribution function

$$
\begin{gathered}
N_{0}\left(p \geq p_{0}\right)=A_{0} p\left[1+\left(\frac{p}{p_{c}}\right)^{2}\right]^{-\rho}, A_{0}=\frac{4 \pi S_{0} \psi p_{0}^{2}}{U_{2} \Gamma_{2}\left(1+3 \beta_{1}^{2}\right) p_{c}^{2}}\left[1+\frac{p_{0}^{2}}{p_{c}^{2}}\right]^{\rho-1} \\
\rho=\frac{\psi}{2\left(1+3 \beta_{1}^{2}\right)}+1,
\end{gathered}
$$

illustrated in Fig. 1.

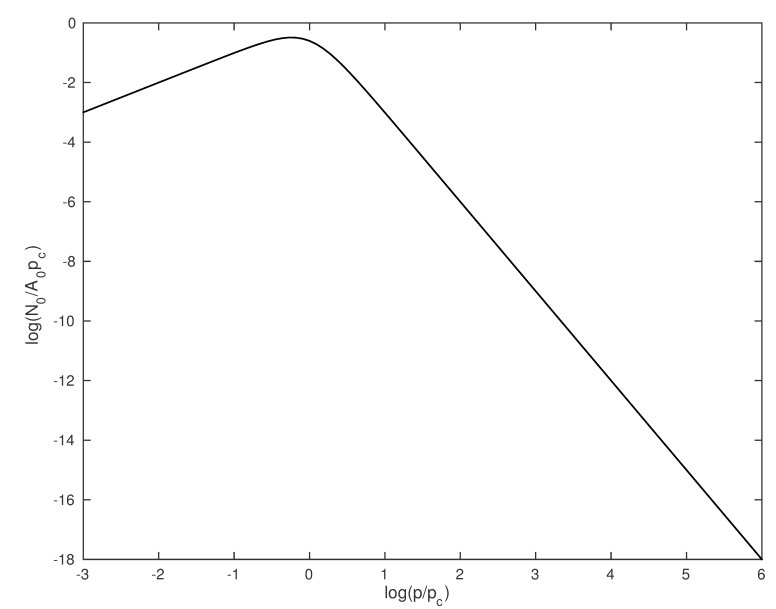

Figure 1: Differential number density of accelerated particles at the shock as a function of $p / p_{c}$ in the case $R=0$ for the adopted spectral index value $\rho=2$ and injection momentum $p_{0} / p_{c}=10^{-3}$.

For particle momenta $p_{0} \leq p \leq p_{c}$ the Lorentzian distribution (4.2) increases linearly with momentum, $N_{0}\left(p_{0} \leq p \leq p_{c}\right) \simeq A_{0} p$, whereas for large momenta $p \geq p_{c}$ it approaches the decreasing power law distribution

$$
N_{0}\left(p \geq p_{c}\right) \simeq A_{0} p_{c}\left(\frac{p}{p_{c}}\right)^{-\xi}, \xi=2 \rho-1=1+\frac{\psi}{1+3 \beta_{1}^{2}}
$$


Notice that for relativistic $\left(\Gamma_{1} \gg 1\right)$ shock speeds the characteristic momentum $p_{c}$ coincides with $m c$, so that in this case no decreasing power law distributions for nonrelativistic shock accelerated particles result.

For nonrelativistic shock velocities $\beta_{1} \ll 1$, the Lorentzian distribution function (4.2) reads

$$
N_{0}\left(p \geq p_{0}\right)=A_{0} p\left[1+\left(\frac{p}{\sqrt{3} \beta_{1} m c}\right)^{2}\right]^{-\frac{2 r+1}{2(r-1)}}
$$

At momenta greater than the nonrelativistic characteristic momentum $p_{c}^{\mathrm{nr}}=\sqrt{3} m U_{1}$ this function approaches the decreasing power law distribution $N_{0}\left(p \geq p_{c}^{\mathrm{nr}}\right) \simeq A_{0} p_{c}^{\mathrm{nr}}\left(\frac{p}{p_{c}}\right)^{-\xi_{0}}$ with the spectral index $\xi_{0}=(r+2) /(r-1)$ agreeing with the standard result for nonrelativistic shocks.

For relativistic shock velocities with $\beta_{1} \simeq 1$ and $\Gamma_{1} \gg 1$, we have to distinguish between particle injection at nonrelativistic $\left(p_{0} \ll m c\right)$ and at relativistic $\left(p_{0} \gg m c\right)$ momenta. In the first case the Lorentzian distribution function (4.2) reads

$$
N_{0}\left(p \geq p_{0}\right)=A_{0} p\left[1+\left(\frac{p}{m c}\right)^{2}\right]^{-1-\frac{3}{8\left(\Gamma_{1} \sqrt{r^{2}-1}-1\right)}}
$$

At nonrelativistic particle momenta this function increases linearly in momentum. At relativistic particle momenta it approaches the decreasing power law distribution

$$
N_{0}(p \geq m c) \simeq A_{0} m c\left(\frac{p}{m c}\right)^{-\xi}, \xi=1+\frac{3}{4\left(\Gamma_{1} \sqrt{r^{2}-1}-1\right)}
$$

If cosmic rays are injected at relativistic momenta the power law behavior (4.6) holds throughout for $p \geq p_{0} \gg m c$

In the case of an ultrarelativistic hydrodynamical shock $\Gamma_{1} \gg 1$ and a relativistic downstream medium with adiabatic index 4/3, the flow compression ratio is $r \simeq 3$ (Blandford and McKee 1976), so that the power law spectral index (4.6) becomes

$$
\xi=1+\frac{3}{\left[\sqrt{8 \Gamma_{1}^{2}+1}-1\right]\left[4-\frac{3}{\Gamma_{1}^{2}}\right]} \simeq 1+\frac{3}{8 \sqrt{2} \Gamma_{1}},
$$

illustrated in Fig. 2 for relativistic shocks with $\Gamma_{1} \geq 2$.

Obviously relativistic shocks accelerate cosmic ray particles very efficiently in the case of negligible momentum losses to power law distribution functions at relativistic momenta with power law spectral index values close to unity due to the dominating $\Gamma^{-1}$ dependence of $\xi-1$.

Our result of efficient cosmic ray acceleration with flat power-law spectral indices $\xi \simeq 1$ for ultrarelativistic shocks disagrees strongly with the earlier established universal spectral index value $\xi \in[2.25-2.30]$ from the eigenfunction and Monte Carlo simulation studies[13]. As possible explanation for this difference we recall that our analytical solution is based on two continuity conditions at the shock. These two continuity conditions are needed as our steady-state diffusionconvection transport equation (3.2) is a second-order differential equation in the position coordinate $z$. While the continuity condition for the particle phase density at the shock is also used in the eigenfunction solution method, the continuity condition for the flux of particles is not used in that method as the Fokker-Planck transport equation (2.1) is a first-order differential equation in the 


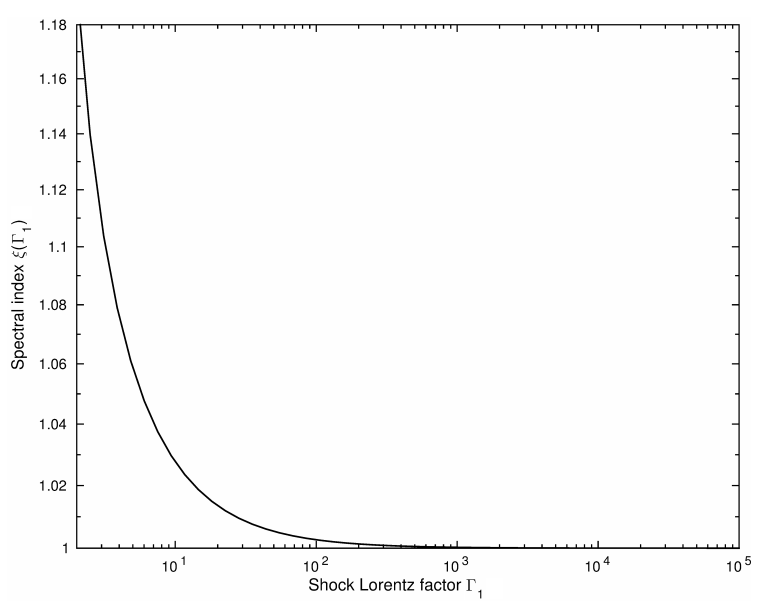

Figure 2: Power law spectral index of relativistic particles accelerated at an ultrarelativistic shock for the case $R=0$ as a function of the shock Lorentz factor $\Gamma_{1}$.

position coordinate $z$. It is clear that the use of different continuity conditions results in different results.

Finally we emphasize that the isotropic differential number densities (3.8) and (4.2) - (4.6) hold in the mixed comoving coordinate system. Transforming the momentum coordinate $p$ to the observer's frame yields highly anisotropic particle number densities in the case of relativistic shock speeds.

\section{References}

[1] Drury, L. O. C., 1983, Rep. Progr. Phys. 46, 973

[2] Blandford, R., Eichler, D., 1987, Phys. Rep. 154, 1

[3] Schlickeiser, R., 2011, ApJ 732, 96

[4] Axford, W. I., Leer, E., Skadron, G., 1977, Proc. 15th Intern. Cosmic Ray Conf., Plovdiv, Bulgaria, Vol. 11, p. 132

[5] Krymskii, G. F., 1077, Sov. Phys. Doklady 22, 327

[6] Blandford, R., Ostriker, J. P., 1978, ApJ 221, L29

[7] Bell, A. R., 1978, MNRAS 182, 147

[8] Webb, G. M., 1985, ApJ 296, 319

[9] Kirk,J. G., Schneider, P., Schlickeiser, 1988, ApJ 328, 269

[10] Riffert, H., 1986, ApJ 310, 729

[11] Schlickeiser, R., 2015, ApJ, submitted (arXiv: :1503.04737)

[12] Blandford, R., McKee, C. F., 1976, Phys. Fluids 19, 1130

[13] Kirk,J. G., Duffy, P., 1999, J. Phys. G 25, R163 